\title{
Novel Stereoselective Syntheses of Highly Functionalized Benzannulated \\ Pyrrolizidines and Indolizidines by Samarium Diiodide-Induced \\ Cyclizations of Indole Derivatives
}

\author{
Steffen Gross and Hans-Ulrich Reissig* \\ Institut für Chemie - Organische Chemie, Freie Universität Berlin, Takustr. 3, D-14195 \\ Berlin, Germany \\ hans.reissig@chemie.fu-berlin.de
}

Experimental Conditions: All reactions were performed under argon atmosphere in flame dried flasks. Unless otherwise stated, materials were obtained from commercial suppliers and were used without further purification. Hexamethylphosphoramide was distilled and kept under argon. Tetrahydrofuran was freshly distilled from sodium/benzophenone under argon for each of the $\mathrm{SmI}_{2}$ reactions.

General experimental procedure: Samarium (2.4 - 2.5 equiv) and 1,2-diiodoethane (2.2 equiv) were suspended in freshly distilled anhydrous THF (10 mL per mmol samarium) under an argon atmosphere and stirred for $2 \mathrm{~h}$ at room temperature. To the resulting dark blue solution HMPA (10 equiv.) was added if necessary. The ketone and phenol (2.0 equiv.), dissolved in THF $(20 \mathrm{~mL})$, were then added in one portion to the deep blue solution. After 16 $\mathrm{h}$ the reaction was quenched with saturated aqueous solution of sodium bicarbonate, the organic layer was separated and the aqueous layer was extracted with diethyl ether $(3 \times 25$ $\mathrm{mL})$. The combined ether extracts were washed with brine $(25 \mathrm{~mL})$, dried over anhydrous magnesium sulfate, filtered and evaporated. The resulting crude product was purified by flash chromatography on silica gel using hexane/ethyl acetate mixtures.

\section{4-(9H-Carbazol-9-yl)butan-2-ol (2)}

Analytical data: colorless solid; $\mathrm{mp} 84-88{ }^{\circ} \mathrm{C} ;{ }^{1} \mathrm{H}$ NMR $\left(\mathrm{CDCl}_{3}, 270 \mathrm{MHz}\right): \delta=1.15$ (d, $J=$ $5.9 \mathrm{~Hz}, 3 \mathrm{H}, 1-\mathrm{H}), 1.64$ (s, br, 1H, OH), 1.76-1.91 (m, 1H, 3-H), 1.96-2.08 (m, 1H, 3-H), 3.72 $\left(\mathrm{m}_{\mathrm{c}}, 1 \mathrm{H}, 2-\mathrm{H}\right), 4.36$ (ddd, $\left.J=4.6,7.3,14.9 \mathrm{~Hz}, 1 \mathrm{H}, 4-\mathrm{H}\right), 4.50$ (ddd, $J=6.6,8.5,14.9 \mathrm{~Hz}, 1 \mathrm{H}$, $1-\mathrm{H}), 7.25\left(\mathrm{~m}_{\mathrm{c}}, 2 \mathrm{H}, \mathrm{Ar}\right), 7.48(\mathrm{~d}, J=3.9 \mathrm{~Hz}, 4 \mathrm{H}, \mathrm{Ar}), 8.12(\mathrm{~d}, J=7.8 \mathrm{~Hz}, 2 \mathrm{H}, \mathrm{Ar}) ;{ }^{13} \mathrm{C} \mathrm{NMR}$ $\left(\mathrm{CDCl}_{3}, 68 \mathrm{MHz}\right): \delta=24.12$ (q, C-1), 37.6, 39.4 (2t, C-3, C-4), 65.2 (d, C-2), 108.6, 118.7, 120.3 (3d, Ar), 122.7 (s, $\mathrm{C}_{\mathrm{ipso}}$ ), 125.6 (d, Ar), 140.3 (s, $\mathrm{C}_{\mathrm{ipso}}$ ); IR (Film): v = $3345 \mathrm{~cm}^{-1}(\mathrm{OH})$, 3045 (ArH), 2970-2875 (CH), $1595(\mathrm{C}=\mathrm{C})$; MS (EI, $\left.120^{\circ} \mathrm{C}, 80 \mathrm{eV}\right): \mathrm{m} / \mathrm{z}(\%)=239\left[\mathrm{M}^{+}\right](43)$, 
$194\left[\mathrm{M}^{+}-\mathrm{C}_{2} \mathrm{H}_{5} \mathrm{O}\right](1), 180\left[\mathrm{M}^{+}-\mathrm{C}_{3} \mathrm{H}_{7} \mathrm{O}\right](100)$; HRMS $\left(120{ }^{\circ} \mathrm{C}, 80 \mathrm{eV}\right):\left[\mathrm{M}^{+}\right]: \mathrm{C}_{16} \mathrm{H}_{17} \mathrm{NO}$ calcd.: 239.13101; found: 239.13066; $\mathrm{C}_{16} \mathrm{H}_{17} \mathrm{NO}$ (239.3) calcd.: $\mathrm{C}$ 80.41, H 7.17, N 5.86; found: C 79.72, H 6.64, N 5.57.

$\left(1 R^{*}, 9 \mathrm{a} S^{*}\right)-1-M e t h y l-2,3,9,9 a-t e t r a h y d r o-1 H$-pyrrolo[1,2-a]indol-1-ol (5)

Analytical data: colorless oil; ${ }^{1} \mathrm{H}$ NMR $\left(\mathrm{CDCl}_{3}, 500 \mathrm{MHz}\right): \delta=1.04\left(\mathrm{~s}, 3 \mathrm{H}, 1-\mathrm{CH}_{3}\right), 1.81$ (ddd, $J=4.3,8.0,12.3 \mathrm{~Hz}, 1 \mathrm{H}, 2-\mathrm{H}), 2.04(\mathrm{td}, J=8.0,12.3 \mathrm{~Hz}, 1 \mathrm{H}, 2-\mathrm{H}), 2.84$ (s, br, 1H, $\mathrm{OH}), 3.05-3.10(\mathrm{~m}, 3 \mathrm{H}, 9-\mathrm{H}, 3-\mathrm{H}), 3.60$ (ddd, $J=4.2,8.0,10.6 \mathrm{~Hz}, 1 \mathrm{H}, 3-\mathrm{H}), 3.91$ (dd, $J=$ 5.6, 8.1 Hz, 1H, 9a-H), 6.56 (d, $J=7.8 \mathrm{~Hz}, 1 \mathrm{H}, 5-\mathrm{H}), 6.74$ (dt, $J=1.0,7.3 \mathrm{~Hz}, 1 \mathrm{H}, 7-\mathrm{H}), 7.04$ $\left(\mathrm{d}^{*} J=7.4 \mathrm{~Hz}, 1 \mathrm{H}, 8-\mathrm{H}\right), 7.08(\mathrm{t}, J=7.8 \mathrm{~Hz}, 1 \mathrm{H}, 6-\mathrm{H}) ;{ }^{*}$ with further splitting; ${ }^{13} \mathrm{C}$ NMR $\left(\mathrm{CDCl}_{3}, 125 \mathrm{MHz}\right): \delta=21.9$ (q, 1-CH $), 29.5,41.1,50.5$ (3t, C-9, C-2, C-3), 72.9 (d, C-9a), 77.3 (s, C-1), 109.9, 119.5, 124.1, 127.5, 129.9 (4d, C-5, C-7, C-8, C-6), 129.9, 154.0 (2s, $\mathrm{C}_{\text {ipso }}$ ); IR (film): $v=3365 \mathrm{~cm}^{-1}(\mathrm{OH}), 3070-3025$ (=CH), 2970-2870 (CH), 1605 (C=C); MS $\left(\mathrm{EI}, 100{ }^{\circ} \mathrm{C}, 80 \mathrm{eV}\right): \mathrm{m} / z(\%)=189\left[\mathrm{M}^{+}\right](57), 169\left[\mathrm{M}^{+}-\mathrm{H}_{2}-\mathrm{H}_{2} \mathrm{O}\right](16), 154$ (37), 131 (100); $\mathrm{C}_{12} \mathrm{H}_{15} \mathrm{NO}$ (189.3) calcd.: C 76.16, H 7.99, N 7.40; found: C 75.90, H 8.14, N 7.33.

\section{$\left(9 R^{*}, 9 \mathrm{a} S^{*}\right)-9-M e t h y l-6,7,8,9,9 \mathrm{a}, 10-h e x a h y d r o p y r i d o[1,2-a]$ indol-9-ol (6)}

Analytical data: colorless oil; ${ }^{1} \mathrm{H}$ NMR $\left(\mathrm{CDCl}_{3}, 500 \mathrm{MHz}\right): \delta=1.30\left(\mathrm{~s}, 3 \mathrm{H}, 9-\mathrm{CH}_{3}\right), 1.46(\mathrm{dt}$, $J=5.1,12.6 \mathrm{~Hz}, 1 \mathrm{H}, 8-\mathrm{H}), 1.60(\mathrm{~s}, \mathrm{br}, 1 \mathrm{H}, \mathrm{OH}), 1.68-1.80(\mathrm{~m}, 2 \mathrm{H}, 7-\mathrm{H}), 1.93\left(\mathrm{td}^{*}, J=4.0\right.$, $12.6 \mathrm{~Hz}, 1 \mathrm{H}, 8-\mathrm{H}), 2.47\left(\mathrm{dt}^{*}, J=4.0,11.5 \mathrm{~Hz}, 1 \mathrm{H}, 6-\mathrm{H}\right), 2.81\left(\mathrm{dd}^{*}, J=12.1,15.2 \mathrm{~Hz}, 1 \mathrm{H}, 10-\right.$ H), 2.94 (dd, $J=7.9,15.2 \mathrm{~Hz}, 1 \mathrm{H}, 10-\mathrm{H}), 3.08$ (dd, $J=7.9,12.1 \mathrm{~Hz}, 1 \mathrm{H}, 9 \mathrm{a}-\mathrm{H}), 3.58\left(\mathrm{dd}^{*}, J=\right.$ 4.8, 11.5 Hz, 1H, 6-H), 6.45 (d, $J=7.8 \mathrm{~Hz}, 1 \mathrm{H}, 4-\mathrm{H}), 6.68$ (dt, $J=1.0,7.2 \mathrm{~Hz}, 1 \mathrm{H}, 2-\mathrm{H}), 7.07$ (t, br, $J=7.8 \mathrm{~Hz}, 1 \mathrm{H}, 3-\mathrm{H}), 7.11\left(\mathrm{~d}^{*}, J=7.2 \mathrm{~Hz}, 1 \mathrm{H}, 1-\mathrm{H}\right) ;{ }^{*}$ with further splitting; ${ }^{13} \mathrm{C}$ NMR $\left(\mathrm{CDCl}_{3}, 125 \mathrm{MHz}\right): \delta=20.9$ (q, 9-CH $\left.\mathrm{CH}_{3}\right), 23.5,29.4,40.8,45.1$ (4t, C-7, C-10, C-8, C-6), 71.0 (s, C-9), 73.7 (d, C-9a), 106.6, 118.1, 124.6, 127.3 (4d, C-4, C-2, C-3, C-1), 128.7, 151.7 (2s, $\mathrm{C}_{\text {ipso }}$ ); IR (film): $v=3430 \mathrm{~cm}^{-1}(\mathrm{OH}), 3050-3025$ (=CH), 2940-2690 (CH), 1610 (C=C); MS $\left(\mathrm{EI}, 100{ }^{\circ} \mathrm{C}, 80 \mathrm{eV}\right): \mathrm{m} / z(\%)=203\left[\mathrm{M}^{+}\right](46), 186\left[\mathrm{M}^{+}-\mathrm{OH}\right](4), 170\left[\mathrm{M}^{+}-\mathrm{CH}_{3}-\mathrm{H}_{2} \mathrm{O}\right](5)$, 145 (11), 132 [Indole-N-CH${ }_{3}^{+}$] (100); $\mathrm{C}_{13} \mathrm{H}_{17} \mathrm{NO}$ (203.3) calcd.: C 76.80, H 8.43, N 6.89; found: C 77.28, H 8.49, N 6.34 . 
$\left(9 R^{*}, 9 \mathrm{a} S^{*}\right)$-9-Hydroxy-9-methyl-8,9,9a,10-tetrahydropyrido[1,2-a]indol-6(7H)-one (9)

Analytical data: colorless solid; mp 152-153 ${ }^{\circ} \mathrm{C} ;{ }^{1} \mathrm{H}$ NMR $\left(\mathrm{CDCl}_{3}, 500 \mathrm{MHz}\right): \delta=1.28$ (s, 3H, 9- $\mathrm{CH}_{3}$ ), 1.95-2.06 (m, 2H, 8-H), 2.53 (ddd, $\left.J=2.8,11.0,18.3 \mathrm{~Hz}, 1 \mathrm{H}, 7-\mathrm{H}\right), 2.69$ (ddd, $J$ $=2.8,7.6,18.3 \mathrm{~Hz}, 1 \mathrm{H}, 7-\mathrm{H}), \mathrm{AB}$-part of ABX-system $\left(\delta_{\mathrm{A}}=3.10, \delta_{\mathrm{B}}=3.14, \mathrm{dd}, J_{\mathrm{AB}}=16.1\right.$ $\left.\mathrm{Hz}, J_{\mathrm{AX}}=9.2 \mathrm{~Hz}, J_{\mathrm{BX}}=9.9 \mathrm{~Hz}, 2 \mathrm{H}, 10-\mathrm{H}\right), 3.39(\mathrm{~s}, \mathrm{br}, 1 \mathrm{H}, \mathrm{OH}), 4.25(\mathrm{dd}, J=9.2,9.9 \mathrm{~Hz}, 1 \mathrm{H}$, 9a-H), 7.04 (dt, $J=1.1,7.6 \mathrm{~Hz}, 1 \mathrm{H}, 1-\mathrm{H}), 7.18\left(\mathrm{~m}_{\mathrm{c}}, 2 \mathrm{H}, 2-\mathrm{H}, 3-\mathrm{H}\right), 8.12$ (d, br, $J=7.9 \mathrm{~Hz}, 1 \mathrm{H}$, 4-H); ${ }^{13} \mathrm{C}-\mathrm{NMR}\left(\mathrm{CDCl}_{3}, 125 \mathrm{MHz}\right): \delta=20.0$ (q, 9- $\left.\mathrm{CH}_{3}\right), 29.9,31.1,36.4$ (3t, C-10, C-7, C-8), 67.5 (d, C-9a), 69.8 (s, C-9), 116.8, 124.3, 124.6, 127.4 (4d, C-4, C-1, C-2, C-3), 129.8, 142.3 (2s, $\mathrm{C}_{\mathrm{ipso}}$ ), 167.8 (s, C-6); IR (film): v = $3315 \mathrm{~cm}^{-1}(\mathrm{OH}), 3070-3040$ (=CH), 2965 (CH), 1625 (CO), $1590(\mathrm{C}=\mathrm{C})$; $\mathrm{MS}\left(\mathrm{EI}, 100{ }^{\circ} \mathrm{C}, 80 \mathrm{eV}\right): \mathrm{m} / \mathrm{z}(\%)=217\left[\mathrm{M}^{+}\right](45), 131$ [Indole- $\mathrm{N}=\mathrm{CH}_{2}^{+}$] (13), 118 [Indole $+\mathrm{H}^{+}$] (100); $\mathrm{C}_{13} \mathrm{H}_{15} \mathrm{NO}_{2}$ (217.3) calcd.: C 71.20, H 6.84, N 6.39; found: $\mathrm{C}$ 71.38, H 6.82, N 6.35 .

\section{$\left(9 R^{*}, 9 \mathrm{a} S *\right)-9-H y d r o x y-8,9,9 a, 10-t e t r a h y d r o p y r i d o[1,2-a]$ indol-6(7H)-one (10)}

Analytical data: colorless solid; mp 191-192 ${ }^{\circ} \mathrm{C}$ (sublimation at $169{ }^{\circ} \mathrm{C}$ ); ${ }^{1} \mathrm{H}$ NMR $\left(\mathrm{CDCl}_{3}\right.$, $500 \mathrm{MHz}): \delta=1.90(\mathrm{dddd}, J=7.3,10.3,10.7,13.1 \mathrm{~Hz}, 1 \mathrm{H}, 8-\mathrm{H}), 2.20\left(\mathrm{~m}_{\mathrm{c}}, 1 \mathrm{H}, 8-\mathrm{H}\right), 2.59$ (ddd, $J=7.3,10.7,18.0 \mathrm{~Hz}, 1 \mathrm{H}, 7-\mathrm{H}), 2.73(\mathrm{ddd}, J=3.1,7.3,18.0 \mathrm{~Hz}, 1 \mathrm{H}, 7-\mathrm{H}), 3.06$ (dd, $J$ $=10.4,15.6 \mathrm{~Hz}, 1 \mathrm{H}, 10-\mathrm{H}), 3.32(\mathrm{dd}, J=8.2,15.6 \mathrm{~Hz}, 1 \mathrm{H}, 10-\mathrm{H}), 3.97$ (ddd, $J=4.5,9.3,10.3$ $\mathrm{Hz}, 1 \mathrm{H}, 9-\mathrm{H}), 4.06$ (ddd, $J=8.2,9.3,10.4 \mathrm{~Hz}, 1 \mathrm{H}, 9 \mathrm{a}-\mathrm{H}), 7.05$ (dt, $J=1.1,7.5 \mathrm{~Hz}, 1 \mathrm{H}, 3-\mathrm{H})$, $7.20\left(\mathrm{~m}_{\mathrm{c}}, 2 \mathrm{H}, 1-\mathrm{H}, 2-\mathrm{H}\right), 8.10\left(\mathrm{~d}^{*}, J=8.6 \mathrm{~Hz}, 1 \mathrm{H}, 4-\mathrm{H}\right)$; ${ }^{*}$ with further splitting; ${ }^{13} \mathrm{C}-\mathrm{NMR}$ $\left(\mathrm{CDCl}_{3}, 125 \mathrm{MHz}\right): \delta=30.1,31.1,34.3$ (3t, C-8, C-7, C-10), 65.4, 71.2 (2d, C-9a, C-9), 117.0, 124.3, 124.6, 127.6 (4d, C-4, C-1, C-2, C-3), 130.1, 142.6, 167.6 (3s, $\left.\mathrm{C}_{\mathrm{ipss}}, \mathrm{C}_{\mathrm{ipso}}, \mathrm{C}-6\right)$; IR (film): $v=3235 \mathrm{~cm}^{-1}(\mathrm{OH}), 2955-2840(\mathrm{CH}), 1625(\mathrm{CO}), 1590(\mathrm{C}=\mathrm{C})$; MS (EI, $100{ }^{\circ} \mathrm{C}, 80$ $\mathrm{eV}): m / z(\%)=203\left[\mathrm{M}^{+}\right](40), 184\left[\mathrm{M}^{+}-\mathrm{H}_{2} \mathrm{O}-\mathrm{H}\right](2), 131$ [Indole- $\left.\mathrm{N}=\mathrm{CH}_{2}^{+}\right]$(11), 118 (100); $\mathrm{C}_{12} \mathrm{H}_{13} \mathrm{NO}_{2}$ (203.2) calcd.: C 70.93, H 6.45, N 6.89; found: C 70.94, H 6.70, N 5.96. 


\section{Methyl $\left(1 R^{*}, 9 \mathrm{a} S^{*}, 9 R^{*}\right)$-1-Hydroxy-1-methyl-2,3,9,9a-tetrahydro-1H-pyrrolo[1,2-}

$a$ ]indol-9-carboxylate (12a) and Methyl $\left(1 S *, 9 \mathrm{a} R *, 9 R^{*}\right)-1-H y d r o x y-1-m e t h y l-2,3,9,9 \mathrm{a}-$

tetrahydro-1H-pyrrolo[1,2-a]indol-9-carboxylate (12b) and Methyl 6-Hydroxy-6-methyl-

5,6-dihydro-4H-pyrrolo[3,2,1-ij]chinolin-1-carboxylate (13)

Analytical data of 12a: colorless oil; ${ }^{1} \mathrm{H}$ NMR $\left(\mathrm{CDCl}_{3}, 500 \mathrm{MHz}\right): \delta=1.06\left(\mathrm{~s}, 3 \mathrm{H}, 1-\mathrm{CH}_{3}\right)$, 1.88 (ddd, $J=3.6,7.3,12.5 \mathrm{~Hz}, 1 \mathrm{H}, 2-\mathrm{H}), 2.16$ (td, $J=8.2,12.5 \mathrm{~Hz}, 1 \mathrm{H}, 2-\mathrm{H}), 2.53$ (s, br, $1 \mathrm{H}$, OH), 3.06 (ddd, $J=7.3,9.1,10.4 \mathrm{~Hz}, 1 \mathrm{H}, 3-\mathrm{H}), 3.66$ (ddd, $J=3.6,8.2,4.5 \mathrm{~Hz}, 1 \mathrm{H}, 3-\mathrm{H}), 3.74$ $\left(\mathrm{s}, 3 \mathrm{H}, \mathrm{OCH}_{3}\right), 4.19$ (d, $\left.J=3.7 \mathrm{~Hz}, 1 \mathrm{H}, 9-\mathrm{H}\right), 4.29$ (d, $\left.J=3.7 \mathrm{~Hz}, 1 \mathrm{H}, 9 \mathrm{a}-\mathrm{H}\right), 6.57$ (dd, $J=0.5$, $7.9 \mathrm{~Hz}, 1 \mathrm{H}, 5-\mathrm{H}), 6.76$ (dt, $J=1.0,7.5, \mathrm{~Hz}, 1 \mathrm{H}, 7-\mathrm{H}), 7.14$ (dddd, $J=0.5,7.5,7.9 \mathrm{~Hz}, 1 \mathrm{H}, 6-$ $\mathrm{H}), 7.23(\mathrm{~d}, \mathrm{br}, J=7.5 \mathrm{~Hz}, 1 \mathrm{H}, 8-\mathrm{H}) ;{ }^{13} \mathrm{C} \mathrm{NMR}\left(\mathrm{CDCl}_{3}, 125 \mathrm{MHz}\right): \delta=21.8\left(\mathrm{q}, 1-\mathrm{CH}_{3}\right), 41.0$ (t, C-2), 47.8 (d, C-9), 50.1 (t, C-3), 52.6 (q, $\mathrm{OCH}_{3}$ ), 75.6 (d, C-9a), 76.8 (s, C-1), 110.3, 119.5, 125.0 (3d, C-5, C-7, C-8), 127.0 (s, C-8a), 129.2 (d, C-6), 153.8, 173.2 (2s, C-4a, CO); IR (film): $v=3410 \mathrm{~cm}^{-1}(\mathrm{OH}), 3070-3000(=\mathrm{CH}), 2970-2875(\mathrm{CH}), 1740(\mathrm{CO}), 1665,1600$ $(\mathrm{C}=\mathrm{C})$; $\mathrm{MS}\left(\mathrm{EI}, 70{ }^{\circ} \mathrm{C}, 80 \mathrm{eV}\right): \mathrm{m} / z(\%)=247\left[\mathrm{M}^{+}\right](35), 189\left[\mathrm{M}^{+}-\mathrm{C}_{3} \mathrm{H}_{6} \mathrm{O}\right)(23), 130$ (100), 84 $\left[\mathrm{M}^{+}-\mathrm{C}_{4} \mathrm{H}_{7} \mathrm{O}\right]$ (93); HRMS (70 $\left.{ }^{\circ} \mathrm{C}, 80 \mathrm{eV}\right):\left[\mathrm{M}^{+}\right]: \mathrm{C}_{14} \mathrm{H}_{17} \mathrm{NO}_{3}$ calcd.: 247.12084; found: 247.12324; $\mathrm{C}_{14} \mathrm{H}_{17} \mathrm{NO}_{3}$ (247.3) calcd.: C 67.99, H 6.93, N 5.66; found: C 67.24, H 6.73, N 5.23 .

Analytical data of 12b: colorless oil; ${ }^{1} \mathrm{H} \mathrm{NMR}\left(\mathrm{CDCl}_{3}, 500 \mathrm{MHz}\right): \delta=0.81\left(\mathrm{~s}, 3 \mathrm{H}, 1-\mathrm{CH}_{3}\right)$, 1.90 (dddd, $J=0.5,1.3,7.5,12.3 \mathrm{~Hz}, 1 \mathrm{H}, 2-\mathrm{H}), 2.25\left(\mathrm{~m}_{\mathrm{c}}, 1 \mathrm{H}, 2-\mathrm{H}\right), 3.04$ (ddd, $J=7.5,10.6$, $11.0 \mathrm{~Hz}, 1 \mathrm{H}, 3-\mathrm{H}), 3.63\left(\mathrm{~m}_{\mathrm{c}}, 1 \mathrm{H}, 3-\mathrm{H}\right), 3.91\left(\mathrm{~s}, 3 \mathrm{H}, \mathrm{OCH}_{3}\right), 4.17(\mathrm{~d}, J=9.7 \mathrm{~Hz}, 1 \mathrm{H}, 9 \mathrm{a}-\mathrm{H})$, 4.50 (d, $J=9.7 \mathrm{~Hz}, 9-\mathrm{H}), 6.52$ (d, $J=7.9 \mathrm{~Hz}, 1 \mathrm{H}, 5-\mathrm{H}), 6.77$ (ddt, $J=0.4,1.0,7.5 \mathrm{~Hz}, 1 \mathrm{H}, 7-$ $\mathrm{H}), 7.15\left(\mathrm{~m}_{\mathrm{c}}, 1 \mathrm{H}, 6-\mathrm{H}\right), 7.43$ (dddd, $\left.J=0.6,1.3,7.5 \mathrm{~Hz}, 1 \mathrm{H}, 8-\mathrm{H}\right) ;{ }^{13} \mathrm{C} \mathrm{NMR}\left(\mathrm{CDCl}_{3}, 125\right.$ MHz): $\delta=22.2\left(\mathrm{q}, 1-\mathrm{CH}_{3}\right), 40.5$ (t, C-2), 47.1 (d, C-9), 49.3 (t, C-3), $52.7\left(\mathrm{q}, \mathrm{OCH}_{3}\right), 73.5$ (d, C-9a), 76.0 (s, C-1), 109.0, 119.0 (2d, C-5, C-7), 124.1 (s, C-8a), 124.7, 128.7 (2d, C-8 C-6), 154.3, 173.0 (2s, C-4a, CO); IR (film): $v=3425 \mathrm{~cm}^{-1}$ (OH), 3050 (=CH), 2950-2900 (CH), 1700 (CO), 1665, $1555(\mathrm{C}=\mathrm{C})$; MS (EI, $\left.110{ }^{\circ} \mathrm{C}, 80 \mathrm{eV}\right): \mathrm{m} / z(\%)=247\left[\mathrm{M}^{+}\right](3), 245\left[\mathrm{M}^{+}-\right.$ $2 \mathrm{H}]$ (28), 198 (100); $\operatorname{HRMS}\left(110{ }^{\circ} \mathrm{C}, 80 \mathrm{eV}\right):\left[\mathrm{M}^{+}-2 \mathrm{H}\right]: \mathrm{C}_{14} \mathrm{H}_{15} \mathrm{NO}_{3}$ calcd.: 245.11869; found: 245.10569.

Analytical data of 13: colorless solid; mp $86{ }^{\circ} \mathrm{C} ;{ }^{1} \mathrm{H} \mathrm{NMR}\left(\mathrm{CDCl}_{3}, 500 \mathrm{MHz}\right): \delta=1.70$ (s, $3 \mathrm{H}, 6-\mathrm{CH}_{3}$ ), 2.66 (ddd, $\left.J=1.7,7.0,12.7 \mathrm{~Hz}, 1 \mathrm{H}, 5-\mathrm{H}\right), 2.86$ (dtd, $J=0.8,9.3,12.7 \mathrm{~Hz}, 1 \mathrm{H}, 5-$ H), 3.97 (s, 3H, $\mathrm{OCH}_{3}$ ), 4.02 (ddd, $\left.J=7.0,9.3,10.7 \mathrm{~Hz}, 1 \mathrm{H}, 4-\mathrm{H}\right), 4.27$ (ddd, $J=1.7,9.3$, $10.7 \mathrm{~Hz}, 1 \mathrm{H}, 4-\mathrm{H}), 5.28$ (s, br, 1H, OH), 7.21-7.27 (m $, 3 \mathrm{H}, 2-\mathrm{H}, 7-\mathrm{H}, 8-\mathrm{H}), 8.00-8.02$ (m, 1H, 
9-H); ${ }^{13} \mathrm{C} \mathrm{NMR}\left(\mathrm{CDCl}_{3}, 125 \mathrm{MHz}\right): \delta=27.1\left(\mathrm{q}, 6-\mathrm{CH}_{3}\right), 42.7(\mathrm{t}, \mathrm{C}-4), 43.4(\mathrm{t}, \mathrm{C}-5), 51.3(\mathrm{q}$, $\mathrm{OCH}_{3}$ ), 75.1 (s, C-6), 98.1 (s, C-1), 110.2 (d, C-2), 121.7, 122.1, 122.4 (3d, C-7, C-8, C-9), 129.8 (s, C-9a), 132.0 (s, C-6a), 157.2 (s, C-9b), 167.3 (s, CO); IR (film): v = 3435 cm$^{-1}$ (OH), $3050(=\mathrm{CH}), 2950-2900(\mathrm{CH}), 1665(\mathrm{CO}), 1615,1555(\mathrm{C}=\mathrm{C})$; MS (EI, $\left.60{ }^{\circ} \mathrm{C}, 80 \mathrm{eV}\right): \mathrm{m} / \mathrm{z}$ $(\%)=245\left[\mathrm{M}^{+}\right](38), 230\left[\mathrm{M}^{+}-\mathrm{CH}_{3}\right](3), 198\left[\mathrm{M}^{+}-\mathrm{CH}_{3}-\mathrm{CH}_{3} \mathrm{OH}\right](100), 170\left[\mathrm{M}^{+}-\mathrm{CH}_{3}-\right.$ $\left.\mathrm{CH}_{3} \mathrm{OH}-\mathrm{CO}_{2}\right](10)$; HRMS $\left(60{ }^{\circ} \mathrm{C}, 80 \mathrm{eV}\right):\left[\mathrm{M}^{+}\right]: \mathrm{C}_{14} \mathrm{H}_{15} \mathrm{NO}_{3}$ calcd.: 245.10519; found: 245.10733; $\mathrm{C}_{14} \mathrm{H}_{15} \mathrm{NO}_{3}$ (245.3) calcd.: $\mathrm{C} 68.55, \mathrm{H} 6.16, \mathrm{~N} 5.71$; found: $\mathrm{C} 69.58, \mathrm{H} \mathrm{5.77,} \mathrm{N}$ 4.83.

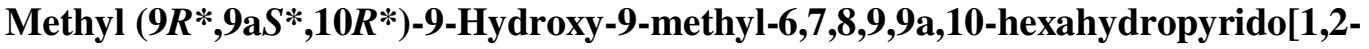
a]indol-10-carboxylate (15)

Analytical data: colorless oil; ${ }^{1} \mathrm{H}$ NMR $\left(\mathrm{CDCl}_{3}, 500 \mathrm{MHz}\right): \delta=1.28\left(\mathrm{~s}, 3 \mathrm{H}, 9-\mathrm{CH}_{3}\right), 1.53$ (dt, $J=4.8,13.0 \mathrm{~Hz}, 1 \mathrm{H}, 8-\mathrm{H}), 1.66-1.81(\mathrm{~m}, 2 \mathrm{H}, 7-\mathrm{H}), 1.98\left(\mathrm{~m}_{\mathrm{c}}, 1 \mathrm{H}, 8-\mathrm{H}\right), 2.55(\mathrm{dt}, J=3.6,11.5$ $\mathrm{Hz}, 1 \mathrm{H}, 6-\mathrm{H}), 2.65$ (s, br, 1H, OH), 3.46 (dd, $J=1.0,11.5 \mathrm{~Hz}, 1 \mathrm{H}, 9 \mathrm{a}-\mathrm{H}), 3.60$ (dd, $J=4.5$, $11.5 \mathrm{~Hz}, 1 \mathrm{H}, 6-\mathrm{H}), 3.84$ (s, 3H, $\mathrm{OCH}_{3}$ ), 4.03 (dd, $\left.J=1.0,11.5 \mathrm{~Hz}, 1 \mathrm{H}, 10-\mathrm{H}\right), 6.49$ (d, $J=7.7$ $\mathrm{Hz}, 1 \mathrm{H}, 4-\mathrm{H}), 6.71$ (tt, $J=1.0,7.5 \mathrm{~Hz}, 1 \mathrm{H}, 2-\mathrm{H}), 7.13$ (qt, $J=1.0,7.7 \mathrm{~Hz}, 1 \mathrm{H}, 3-\mathrm{H}), 7.24$ (m, $1 \mathrm{H}, 1-\mathrm{H}) ;{ }^{13} \mathrm{C} \mathrm{NMR}\left(\mathrm{CDCl}_{3}, 125 \mathrm{MHz}\right): \delta=20.9$ (q, 9-CH $), 23.3$ (t, C-7), 40.7 (t, C-8), 45.0 (t, C-6), 47.3 (d, C-10), 52.6 (q, $\mathrm{OCH}_{3}$ ), 70.4 (s, C-9), 75.2 (d, C-9a), 107.0 (d, C-4), 118.3 (d, C-2), 124.6 (d, C-1), 125.2 (s, C-10a), 128.6 (d, C-3), 150.7 (s, C-4a), 173.5 (s, CO); IR (film): $v=3430 \mathrm{~cm}^{-1}(\mathrm{OH}), 3050(=\mathrm{CH}), 2950-2700(\mathrm{CH}), 1740(\mathrm{CO}), 1665,1605(\mathrm{C}=\mathrm{C})$; MS $\left(\mathrm{EI}, 40{ }^{\circ} \mathrm{C}, 80 \mathrm{eV}\right): \mathrm{m} / \mathrm{z}(\%)=261\left[\mathrm{M}^{+}\right](53), 222(21), 190\left[\mathrm{M}^{+}-\mathrm{C}_{4} \mathrm{H}_{7} \mathrm{O}\right](100) ; \mathrm{HRMS}(40$ $\left.{ }^{\circ} \mathrm{C}, 80 \mathrm{eV}\right):\left[\mathrm{M}^{+}\right]: \mathrm{C}_{15} \mathrm{H}_{19} \mathrm{NO}_{3}$ calcd.: 261.13649; found: 261.13821; $\mathrm{C}_{15} \mathrm{H}_{19} \mathrm{NO}_{3}$ (261.3) calcd.: C 68.90, H 7.33, N 5.36; found: C 68.56, H 7.14, N 5.17.

\section{Methyl $\left(9 R^{*}, 9 \mathrm{a} S^{*}, 10 R^{*}\right)-9-H y d r o x y-9-m e t h y l-6-0 x 0-7,8,9,9 \mathrm{a}, 10-h e x a h y d r o p y r i d o[1,2-$ a]indol-10-carboxylate (17)}

Analytical data : colorless solid; $\mathrm{mp} 58-61{ }^{\circ} \mathrm{C} ;{ }^{1} \mathrm{H}$ NMR $\left(\mathrm{CDCl}_{3}, 500 \mathrm{MHz}\right): \delta=1.24$ (s, 3H, 9- $\mathrm{CH}_{3}$ ), 2.00 (ddd, $\left.J=3.0,8.2,13.0 \mathrm{~Hz}, 1 \mathrm{H}, 8-\mathrm{H}\right), 2.04-2.09$ (m, 1H, 8-H), 2.56 (ddd, $J=8.2$, 10.5, 18.5 Hz, 1H, 7-H), 2.74 (ddd, $J=3.0,7.6,18.5 \mathrm{~Hz}, 1 \mathrm{H}, 7-\mathrm{H}), 2.87$ (s, br, 1H, OH), 3.83 (s, 3H, $\left.\mathrm{OCH}_{3}\right), 4.29$ (d, $\left.J=9.6 \mathrm{~Hz}, 1 \mathrm{H}, 10-\mathrm{H}\right), 4.64$ (d, $\left.J=9.6 \mathrm{~Hz}, 1 \mathrm{H}, 9 \mathrm{a}-\mathrm{H}\right), 7.06$ (dt, $J=$ 1.0, 7.5 Hz, 1H, 3-H), $7.26\left(\mathrm{~m}_{\mathrm{c}}, 1 \mathrm{H}, 2-\mathrm{H}\right), 7.34$ (d, br, J = 7.5 Hz, 1H, 1-H), $8.17(\mathrm{~d}, \mathrm{~J}=8.1$ $\mathrm{Hz}, 1 \mathrm{H}, 4-\mathrm{H}) ;{ }^{13} \mathrm{C} \mathrm{NMR}\left(\mathrm{CDCl}_{3}, 125 \mathrm{MHz}\right): \delta=20.0$ (q, 9- $\left.\mathrm{CH}_{3}\right), 31.1,36.5$ (2t, C-7, C-8), 47.6 (d, C-10), 52.9 (q, $\mathrm{OCH}_{3}$ ), 69.2 (d, C-9a), 69.8 (s, C-9), 117.0, 124.3, 124.4 (3d, C-4, C1, C-3), 126.5 (s, $\mathrm{C}_{\text {ipso }}$ ), 129.0 (d, C-2), 142.0 (s, $\mathrm{C}_{\mathrm{ipso}}$ ), 167.5, $172.2\left(2 \mathrm{~s}, \mathrm{NCO}, \mathrm{CO}_{2} \mathrm{CH}_{3}\right)$; IR 
(film):

$v=3375 \mathrm{~cm}^{-1}(\mathrm{OH}), 3020(=\mathrm{CH}), 2950-2885(\mathrm{CH}), 1745,1635(\mathrm{CO}), 1590(\mathrm{C}=\mathrm{C}) ; \mathrm{MS}(\mathrm{EI}$, $\left.120{ }^{\circ} \mathrm{C}, 80 \mathrm{eV}\right): m / z(\%)=275\left[\mathrm{M}^{+}\right](52), 257\left[\mathrm{M}^{+}-\mathrm{H}_{2} \mathrm{O}\right](53), 198(100), 176(51) ; \mathrm{C}_{15} \mathrm{H}_{17} \mathrm{NO}_{4}$ (275.3) calcd.: C 65.44, H 6.22, N 5.09; found: C 65.09, H 6.20, N 4.95.

\section{Methyl (9R*,9a $\left.S^{*}, 10 R^{*}\right)-10$-Allyl-9-hydroxy-9-methyl-6-oxo-7,8,9,9a,10-hexahydro-} pyrido-[1,2-a]indol-10-carboxylate (18)

Analytical data: colorless oil; ${ }^{1} \mathrm{H}$ NMR $\left(\mathrm{CDCl}_{3}, 500 \mathrm{MHz}\right): \delta=1.20\left(\mathrm{~s}, 3 \mathrm{H}, 9-\mathrm{CH}_{3}\right), 1.74$ (s, br, $1 \mathrm{H}, \mathrm{OH}), 1.86$ (ddd, $J=2.6,7.7,13.1 \mathrm{~Hz}, 1 \mathrm{H}, 8-\mathrm{H}), 1.99$ (dddd, $J=0.9,7.7,13.1 \mathrm{~Hz}, 1 \mathrm{H}$, 8-H), 2.56 (ddd, $J=7.7,11.0,18.5 \mathrm{~Hz}, 1 \mathrm{H}, 7-\mathrm{H}), 2.70$ (ddd, $J=2.6,7.7,18.5 \mathrm{~Hz}, 1 \mathrm{H}, 7-\mathrm{H})$, 2.87

(dd,

$\left.J=9.2,14.2 \mathrm{~Hz}, 1 \mathrm{H}, 10-\mathrm{CH}_{2}\right), 3.02\left(\mathrm{tdd}, J=1.9,5.2,14.2 \mathrm{~Hz}, 1 \mathrm{H}, 10-\mathrm{CH}_{2}\right), 3.69(\mathrm{~s}, 3 \mathrm{H}$, $\left.\mathrm{OCH}_{3}\right), 4.31(\mathrm{~s}, 1 \mathrm{H}, 9 \mathrm{a}-\mathrm{H}), 5.15\left(\mathrm{dtd}, J=0.4,1.5,10.1 \mathrm{~Hz}, 1 \mathrm{H},=\mathrm{CH}_{2}\right), 5.20(\mathrm{dtd}, J=0.9,1.9$, $\left.17.1 \mathrm{~Hz}, 1 \mathrm{H},=\mathrm{CH}_{2}\right), 5.48(\mathrm{dddd}, J=5.2,9.2,10.1,17.1 \mathrm{~Hz}, 1 \mathrm{H},=\mathrm{CH}-), 7.09(\mathrm{dt}, J=1.1,7.6$ $\mathrm{Hz}, 1 \mathrm{H}, 2-\mathrm{H}), 7.21$ (ddd, $J=0.6,1.4,7.7 \mathrm{~Hz}, 1 \mathrm{H}, 1-\mathrm{H}), 7.28$ (ddd, $J=1.4,7.6,8.2 \mathrm{~Hz}, 1 \mathrm{H}, 3-$ $\mathrm{H}), 8.28(\mathrm{ddd}, J=0.6,1.1,8.2 \mathrm{~Hz}, 1 \mathrm{H}, 4-\mathrm{H}) ;{ }^{13} \mathrm{C} \mathrm{NMR}\left(\mathrm{CDCl}_{3}, 125 \mathrm{MHz}\right): \delta=20.3(\mathrm{q}, 9-$ $\mathrm{CH}_{3}$ ), 30.9, 37.8, 44.0 (3t, C-7, C-8, 10-C), 53.1 (q, $\mathrm{OCH}_{3}$ ), 56.6, 70.6 (2s, C-10, C-9), 72.9 (d, C-9a), 116.8 (d, C-4), 121.0 (t, = $\mathrm{CH}_{2}$ ), 124.0, 124.9, 128.9 (3d, C-2, C-1, C-3), 129.7 (s, $\left.\mathrm{C}_{\mathrm{ipso}}\right), 132.2(\mathrm{~d},=\mathrm{CH}-), 142.6\left(\mathrm{~s}, \mathrm{C}_{\mathrm{ipso}}\right), 167.8,175.3\left(2 \mathrm{~s}, \mathrm{NCO}, \mathrm{CO}_{2} \mathrm{CH}_{3}\right)$; IR (film): $v=3430$ $\mathrm{cm}^{-1}(\mathrm{OH}), 3075(=\mathrm{CH}), 2975-2885(\mathrm{CH}), 1730,1660(\mathrm{CO}), 1595(\mathrm{C}=\mathrm{C})$; MS (EI, $100{ }^{\circ} \mathrm{C}, 80$ $\mathrm{eV}): m / z(\%)=315\left[\mathrm{M}^{+}\right](33), 274\left[\mathrm{M}^{+}-\mathrm{CHO}\right](5), 176(100) ; \mathrm{C}_{15} \mathrm{H}_{17} \mathrm{NO}_{4}$ (315.4) calcd.: $\mathrm{C}$ 68.55, H 6.71, N 4.44; found: C 68.34, H 6.67, N 4.28.

\section{Methyl $\left(9 R^{*}, 9 \mathrm{a} S^{*}, 10 R^{*}\right)-10-A l l y l-9-\{[$ tert-butyl(dimethyl)siloxy]ethyl\}-9-hydroxy-6-oxo-} 6,7,8,9,9a,10-hexahydropyrido[1,2-a]indol-10-carboxylate (20)

Analytical data: colorless crystals; mp $127-131{ }^{\circ} \mathrm{C} ;{ }^{1} \mathrm{H}$ NMR $\left(\mathrm{CDCl}_{3}, 500 \mathrm{MHz}\right): \delta=0.05$ (s, 6H, Si-CH $)_{3}$ ), 0.86 [s 9H, C( $\left.\left.\mathrm{CH}_{3}\right)_{3}\right], 1.64$ (td, $\left.J=3.8,14.6, \mathrm{~Hz}, 1 \mathrm{H}, 9-\mathrm{CH}_{2}\right), 1.79$ (ddd, $J=$ 5.5, 9.7, $\left.14.6 \mathrm{~Hz}, 1 \mathrm{H}, 9-\mathrm{CH}_{2}\right), 1.85-1.88(\mathrm{~m}, 1 \mathrm{H}, 8-\mathrm{H}), 2.16$ (ddd, $J=3.5,7.0,13.8 \mathrm{~Hz}, 1 \mathrm{H}, 8-$ H), 2.46 (ddd, $J=7.0,10.9,18.1 \mathrm{~Hz}, 1 \mathrm{H}, 7-\mathrm{H}), 2.69$ (ddd, $J=3.5,7.6,18.1 \mathrm{~Hz}, 1 \mathrm{H}, 7-\mathrm{H})$, $2.93\left(\mathrm{dd}, J=9.0,14.3 \mathrm{~Hz}, 1 \mathrm{H}, 10-\mathrm{CH}_{2}\right), 3.02\left(\mathrm{tdd}, J=1.5,5.5,14.3 \mathrm{~Hz}, 1 \mathrm{H}, 10-\mathrm{CH}_{2}\right), 3.60$ (s, $\left.3 \mathrm{H}, \mathrm{OCH}_{3}\right), 3.77-3.87\left(\mathrm{~m}, 2 \mathrm{H}, \mathrm{OCH}_{2}\right), 4.28(\mathrm{~s}, 1 \mathrm{H}, 9 \mathrm{a}-\mathrm{H}), 4.34(\mathrm{~s}, 1 \mathrm{H}, \mathrm{OH}), 5.08(\mathrm{~d}, J=10.2$ $\left.\mathrm{Hz}, 1 \mathrm{H},=\mathrm{CH}_{2}\right), 5.14\left(\mathrm{~d}, \mathrm{~J}=17.0 \mathrm{~Hz}, 1 \mathrm{H},=\mathrm{CH}_{2}\right), 5.49(\mathrm{ddd}, J=5.5,9.0,10.2,17.0 \mathrm{~Hz}, 1 \mathrm{H}$, $=\mathrm{CH}-), 7.05$ (dt, $J=1.1,7.7 \mathrm{~Hz}, 1 \mathrm{H}, 1-\mathrm{H}), 7.13(\mathrm{dd}, J=1.4,7.8 \mathrm{~Hz}, 1 \mathrm{H}, 3-\mathrm{H}), 7.24$ (dt, $J=$ 
1.1, 7.7 Hz, 1H, 2-H), $8.22(\mathrm{~d}, J=7.8 \mathrm{~Hz}, 1 \mathrm{H}, 4-\mathrm{H}) ;{ }^{13} \mathrm{C} \mathrm{NMR}\left(\mathrm{CDCl}_{3}, 125 \mathrm{MHz}\right): \delta=-5.7$, $5.6\left(2 \mathrm{~s}, \mathrm{Si}-\mathrm{CH}_{3}\right), 18.0,25.7$ [s, q, C( $\left.\mathrm{CH}_{3}\right)_{3}$ ], 30.9, 31.7, 33.1, 41.4 (4t, 9-C, C-7, C-8, 10-C), $52.3\left(\mathrm{q}, \mathrm{OCH}_{3}\right), 55.9$ (s, C-10), $59.7\left(\mathrm{t}, \mathrm{OCH}_{2}\right), 73.0$ (d, C-9a), 73.2 (s, C-9), 116.8 (d, C-4), $120.5\left(\mathrm{t},=\mathrm{CH}_{2}\right), 124.0,124.1,128.7$ (3d, C-1, C-3, C-2), $131.1\left(\mathrm{~s}, \mathrm{C}_{\mathrm{ipso}}\right), 132.7$ (d, =CH-), 142.7, 168.1, 173.7 (s, $\left.\mathrm{C}_{\mathrm{ipso}}, \mathrm{NCO}, \mathrm{CO}_{2} \mathrm{CH}_{3}\right)$; IR (KBr): $v=3455 \mathrm{~cm}^{-1}(\mathrm{OH}), 3065(=\mathrm{CH})$, 2955-2855 (CH), 1730, $1665(\mathrm{CO}), 1595(\mathrm{C}=\mathrm{C})$; MS (EI, $\left.135^{\circ} \mathrm{C}, 80 \mathrm{eV}\right): \mathrm{m} / \mathrm{z}(\%)=459$ $\left[\mathrm{M}^{+}\right]$(16), $402\left[\mathrm{M}^{+}-\mathrm{C}_{3} \mathrm{H}_{5} \mathrm{O}\right]$ (99), 286 (90), 144 (86), 111 (100); $\mathrm{C}_{25} \mathrm{H}_{37} \mathrm{NO}_{5} \mathrm{Si}$ (445.7) calcd.: C 65.33, H 8.04, N 3.05; found: C 65.16, H 8.11, N 2.88.

\section{Methyl (2a $\left.R^{*}, 10 \mathrm{~b} S^{*}, 10 \mathrm{c} R^{*}\right)-2 \mathrm{a}-H y d r o x y-5-o x o-1,2,2 \mathrm{a}, 4,5,10 \mathrm{c}-h$ exahydrobenzo[b]penta-} cyclo[hi]indol-10b(3H)-carboxylate (22)

Analytical data: colorless solid; mp $167-169{ }^{\circ} \mathrm{C}$ (sublimation at $150{ }^{\circ} \mathrm{C}$ ); ${ }^{1} \mathrm{H}$ NMR $\left(\mathrm{CDCl}_{3}\right.$, $500 \mathrm{MHz}): \delta=1.64(\mathrm{ddd}, J=7.4,12.8,13.0 \mathrm{~Hz}, 1 \mathrm{H}, 1-\mathrm{H}), 1.95(\mathrm{dt}, J=4.4,14.1 \mathrm{~Hz}, 1 \mathrm{H}, 3-$ H), 2.06 (dddd, $J=0.6,1.9,6.7,13.7 \mathrm{~Hz}, 1 \mathrm{H}, 1-\mathrm{H}), 2.15$ (ddd, $J=1.9,7.3,13.1 \mathrm{~Hz}, 1 \mathrm{H}, 2-\mathrm{H})$, 2.21 (ddd, $J=2.6,5.7,14.6 \mathrm{~Hz}, 1 \mathrm{H}, 3-\mathrm{H}), 2.44$ (dddd, $J=0.5,2.6,4.1,14.6 \mathrm{~Hz}, 1 \mathrm{H}, 4-\mathrm{H}$ ), $2.71\left(\mathrm{~m}_{\mathrm{c}}, 2 \mathrm{H}, 2-\mathrm{H}, 4-\mathrm{H}\right), 3.00(\mathrm{~s}, 1 \mathrm{H}, \mathrm{OH}), 3.80\left(\mathrm{~s}, 3 \mathrm{H}, \mathrm{OCH}_{3}\right), 4.75(\mathrm{~d}, J=1.6 \mathrm{~Hz}, 1 \mathrm{H}, 10 \mathrm{c}-$ H), 7.09 (ddt, $J=0.7,1.1,7.6 \mathrm{~Hz}, 1 \mathrm{H}, 8-\mathrm{H}), 7.28\left(\mathrm{t}^{*}, J=8.1 \mathrm{~Hz}, 1 \mathrm{H}, 9-\mathrm{H}\right), 7.43\left(\mathrm{~d}^{*}, J=7.6\right.$ $\mathrm{Hz}, 1 \mathrm{H}, 10-\mathrm{H}), 8.05\left(\mathrm{~d}^{*}, J=8.1 \mathrm{~Hz}, 1 \mathrm{H}, 7-\mathrm{H}\right)$; with further splitting; ${ }^{13} \mathrm{C} \mathrm{NMR}\left(\mathrm{CDCl}_{3}, 125\right.$ MHz): $\delta=32.8,33.7,39.0,39.6$ (4t, C-4, C-3, C-2, C-1), 53.0 (q, $\mathrm{OCH}_{3}$ ), 61.6 (s, C-10b), 75.8 (d, C-10c), 81.7 (s, C-2a), 115.6, 124.3, 124.4, 129.2 (4d, Ar), 131.5, 141.7, 170.6, 174.0 (4s, C-10a, C-6a, C-5, $\mathrm{CO}_{2} \mathrm{CH}_{3}$ ); IR (film): $v=3300 \mathrm{~cm}^{-1}(\mathrm{OH}), 3080$ (=CH), 2975-2855 $(\mathrm{CH}), 1730,1640(\mathrm{CO}), 1590(\mathrm{C}=\mathrm{C}) ; \mathrm{MS}\left(\mathrm{EI}, 140{ }^{\circ} \mathrm{C}, 80 \mathrm{eV}\right): \mathrm{m} / z(\%)=287\left[\mathrm{M}^{+}\right](61), 269$ $\left[\mathrm{M}^{+}-\mathrm{H}_{2} \mathrm{O}\right](10), 228\left[\mathrm{M}^{+}-\mathrm{CH}_{3}-\mathrm{CO}_{2}\right]$ (4), $210\left[\mathrm{M}^{+}-\mathrm{H}_{2} \mathrm{O}-\mathrm{CH}_{3}-\mathrm{CO}_{2}\right](100) ; \mathrm{HRMS}\left(140{ }^{\circ} \mathrm{C}\right.$, $80 \mathrm{eV}$ ): $\left[\mathrm{M}^{+}\right]: \mathrm{C}_{16} \mathrm{H}_{17} \mathrm{NO}_{4}$ calcd.: 287.11576; found: 287.11734 .

\section{Diethyl $\left(1 R^{*}, 7 R^{*}, 7 \mathrm{a} S^{*}\right)-7-H y d r o x y-7-m e t h y l-5,6,7,7 \mathrm{a}-$ tetrahydro-1H-pyrrolizin-1,2-} dicarboxylate (25) and Diethyl 7-Hydroxy-7-methyl-5,6,7,7a-tetrahydro-1H-pyrrolizin1,2-dicarboxylate (mixture of minor stereo isomers)

Analytical data of 25: yellow oil; ${ }^{1} \mathrm{H}$ NMR $\left(\mathrm{CDCl}_{3}, 500 \mathrm{MHz}\right): \delta=1.16,1.18(2 \mathrm{t}, J=7.1$ $\mathrm{Hz}$, je $3 \mathrm{H}, \mathrm{CH}_{2} \mathrm{CH}_{3}$ ), 1.21 (s, 3H, 7- $\left.\mathrm{CH}_{3}\right), 1.76$ (ddd, $\left.J=5.3,7.5,12.7 \mathrm{~Hz}, 1 \mathrm{H}, 6-\mathrm{H}\right), 1.99$ (td, $J=8.0,12.7 \mathrm{~Hz}, 1 \mathrm{H}, 6-\mathrm{H}), 3.13-3.18(\mathrm{~m}, 1 \mathrm{H}, 5-\mathrm{H}), 3.35$ (ddd, $J=5.3,8.0,10.8 \mathrm{~Hz}, 1 \mathrm{H}, 5-\mathrm{H})$, 3.87 (dd, $J=1.0,4.5 \mathrm{~Hz}, 1 \mathrm{H}, 1-\mathrm{H}), 3.90$ (d, $J=4.5 \mathrm{~Hz}, 1 \mathrm{H}, 7 \mathrm{a}-\mathrm{H}), 4.04,4.10(2 \mathrm{q}, J=7.1 \mathrm{~Hz}$, je $\left.2 \mathrm{H}, \mathrm{CH}_{2} \mathrm{CH}_{3}\right), 7.00(\mathrm{~d}, J=1.0, \mathrm{~Hz}, 1 \mathrm{H}, 3-\mathrm{H}) ;{ }^{13} \mathrm{C} \mathrm{NMR}\left(\mathrm{CDCl}_{3}, 125 \mathrm{MHz}\right): \delta=14.0,14.3$ 
(2q, $\mathrm{CH}_{2} \mathrm{CH}_{3}$ ), 22.2 (q, 7- $\mathrm{CH}_{3}$ ), 40.4 (t, C-6), 46.9 (t, C-5), 47.5 (d, C-1), 59.3, 61.1 (2t, $\mathrm{OCH}_{2}$ ), 76.7 (s, C-7), 77.9 (d, C-7a), 105.4 (s, C-2), 152.7 (d, C-3), 165.0, 174.2 (2s, CO); IR (film): $v=3445 \mathrm{~cm}^{-1}(\mathrm{OH}), 2980-2905(\mathrm{CH}), 1735,1690(\mathrm{CO}), 1590(\mathrm{C}=\mathrm{C})$; MS (EI, $120{ }^{\circ} \mathrm{C}$, $80 \mathrm{eV}): m / z(\%)=283\left[\mathrm{M}^{+}\right](22), 238\left[\mathrm{M}^{+}-\mathrm{C}_{2} \mathrm{H}_{5} \mathrm{O}\right](19), 220\left[\mathrm{M}^{+}-\mathrm{C}_{2} \mathrm{H}_{5} \mathrm{O}-\mathrm{H}_{2} \mathrm{O}\right](5), 210$ (56), 164 (100); HRMS (100 $\left.{ }^{\circ} \mathrm{C}, 80 \mathrm{eV}\right):\left[\mathrm{M}^{+}\right]: \mathrm{C}_{14} \mathrm{H}_{21} \mathrm{NO}_{5}$ calcd.: 283.14197; found: 283.14346; $\mathrm{C}_{15} \mathrm{H}_{15} \mathrm{NO}_{4}$ (273.3) calcd.: C 59.35, H 7.47, N 4.94; found: C 59.39, H 7.22, N 4.37 .

Analytical data of minor stereo isomers (yellow oil): Main component: ${ }^{1} \mathrm{H}$ NMR $\left(\mathrm{CDCl}_{3}\right.$, $500 \mathrm{MHz}): \delta=1.21,1.24\left(2 \mathrm{t}, J=7.1 \mathrm{~Hz}\right.$, je $\left.3 \mathrm{H}, \mathrm{CH}_{2} \mathrm{CH}_{3}\right), 1.36\left(\mathrm{~s}, 3 \mathrm{H}, 7-\mathrm{CH}_{3}\right), 1.93(\mathrm{dt}, J=$ 9.9, $13.3 \mathrm{~Hz}, 1 \mathrm{H}, 6-\mathrm{H}), 2.04$ (ddd, $J=2.6,6.6,13.3 \mathrm{~Hz}, 1 \mathrm{H}, 6-\mathrm{H}), 3.33-3.40$ (m, 2H, 5-H), $3.79(\mathrm{~d}, J=4.8 \mathrm{~Hz}, 1 \mathrm{H}, 7 \mathrm{a}-\mathrm{H}), 4.04$ (dd, $J=1.2,4.8 \mathrm{~Hz}, 1 \mathrm{H}, 1-\mathrm{H}), 4.05-4.29$ (m, 4H, $\mathrm{CH}_{2} \mathrm{CH}_{3}$, overlap with signal of the minor component), $7.14(\mathrm{~d}, J=1.2 \mathrm{~Hz}, 1 \mathrm{H}, 3-\mathrm{H}) ;{ }^{13} \mathrm{C}$ NMR $\left(\mathrm{CDCl}_{3}, 125 \mathrm{MHz}\right): \delta=14.1,14.4\left(2 \mathrm{q}, \mathrm{CH}_{2} \mathrm{CH}_{3}\right), 22.6$ (q, 7- $\left.\mathrm{CH}_{3}\right), 39.9$ (t, C-6), 46.1 (d, C-1), 46.6 (t, C-5), 59.3, 61.0 (2t, $\mathrm{OCH}_{2}$ ), 74.2 (s, C-7), 77.6 (d, C-7a), 105.7 (s, C-2), 153.5 (d, C-3), 165.3, 168.6, 169.5, 169.6 (4s, CO for both components).

Minor component: ${ }^{1} \mathrm{H}$ NMR $\left(\mathrm{CDCl}_{3}, 500 \mathrm{MHz}\right): \delta=1.24,1.25(2 \mathrm{t}, J=7.1 \mathrm{~Hz}$, each $3 \mathrm{H}$, $\mathrm{CH}_{2} \mathrm{CH}_{3}$ ), 1.45 (s, 3H, 7- $\mathrm{CH}_{3}$ ), 2.17-2.21 (m, 2H, 6-H), 3.23 (ddd, $J=4.8,7.0,11.7 \mathrm{~Hz}, 1 \mathrm{H}, 5-$ H), 3.67-3.72 (m, 1H, 5-H), 3.65, 4.00 (2d, J = 7.4 Hz, je 1H, 7a-H, 1-H), 4.05-4.29 (m, 4H, $\mathrm{CH}_{2} \mathrm{CH}_{3}$, overlap with signal of the minor component), $7.14(\mathrm{~s}, 1 \mathrm{H}, 3-\mathrm{H}) ;{ }^{13} \mathrm{C} \mathrm{NMR}\left(\mathrm{CDCl}_{3}\right.$, $125 \mathrm{MHz}$ ): $\delta=13.9,14.0$ (2q, $\mathrm{CH}_{2} \mathrm{CH}_{3}$ ), 23.7 (q, 7- $\mathrm{CH}_{3}$ ), 40.2, 40.9 (2t, C-5, C-6), 50.1 (d, C7a), 61.4, 62.8 (2t, $\left.\mathrm{OCH}_{2}\right), 67.2$ (d, C-1), 76.6 (s, C-7), 105.7* (s, C-2), 153.5* (d, C-3), 165.3, 168.6, 169.5, 169.6 (4s, CO for both components). " overlap with signal of the main component. IR (film): $v=3450 \mathrm{~cm}^{-1}(\mathrm{OH}), 2980-2905(\mathrm{CH}), 1735,1690(\mathrm{CO}), 1590(\mathrm{C}=\mathrm{C})$; MS (EI, $\left.120{ }^{\circ} \mathrm{C}, 80 \mathrm{eV}\right): \mathrm{m} / z(\%)=283\left[\mathrm{M}^{+}\right](16), 238\left[\mathrm{M}^{+}-\mathrm{C}_{2} \mathrm{H}_{5} \mathrm{O}\right](12), 220\left[\mathrm{M}^{+}-\mathrm{C}_{2} \mathrm{H}_{5} \mathrm{O}-\right.$ $\left.\mathrm{H}_{2} \mathrm{O}\right](4), 210$ (6), 164 (100); HRMS (120 $\left.{ }^{\circ} \mathrm{C}, 80 \mathrm{eV}\right):\left[\mathrm{M}^{+}\right]: \mathrm{C}_{14} \mathrm{H}_{21} \mathrm{NO}_{5}$ calcd.: 283.14197; found: 283.14355 .

\section{Diethyl $\left(1 R^{*}, 8 R^{*}, 8 \mathrm{a} S^{*}\right)-8$-Hydroxy-8-methyl-1,5,6,7,8,8a-hexahydro-1,2-indolizidindi- carboxylate (26)}

Analytical data: colorless solid; $\mathrm{mp} 93-95^{\circ} \mathrm{C} ;{ }^{1} \mathrm{H}$ NMR $\left(\mathrm{CDCl}_{3}, 500 \mathrm{MHz}\right): \delta=1.14(\mathrm{t}, J=$ $\left.7.1 \mathrm{~Hz}, 3 \mathrm{H}, \mathrm{CH}_{2} \mathrm{CH}_{3}\right), 1.15\left(\mathrm{~s}, 3 \mathrm{H}, 8-\mathrm{CH}_{3}\right), 1.19\left(\mathrm{t}, J=7.1 \mathrm{~Hz}, 3 \mathrm{H}, \mathrm{CH}_{2} \mathrm{CH}_{3}\right), 1.38-1.63(\mathrm{~m}$, $3 \mathrm{H}, 6-\mathrm{H}, 7-\mathrm{H}), 1.79-1.85$ (m, 1H, 7-H), 2.48 (s, 1H, br, OH), 2.84-2.95 (m, 1H, 5-H), 3.33- 
$3.41(\mathrm{~m}, 1 \mathrm{H}, 5-\mathrm{H}), 3.51(\mathrm{~d}, J=8.9 \mathrm{~Hz}, 1 \mathrm{H}, 8 \mathrm{a}-\mathrm{H}), 3.82(\mathrm{dd}, J=1.0,8.9 \mathrm{~Hz}, 1 \mathrm{H}, 1-\mathrm{H}), 4.02$ $\left(\mathrm{m}_{\mathrm{c}}, 2 \mathrm{H}, \mathrm{CH}_{2} \mathrm{CH}_{3}\right), 4.11$ (q, $\left.J=7.1 \mathrm{~Hz}, 2 \mathrm{H}, \mathrm{CH}_{2} \mathrm{CH}_{3}\right), 7.05$ (d, $\left.J=1.0 \mathrm{~Hz}, 1 \mathrm{H}, 3-\mathrm{H}\right) ;{ }^{13} \mathrm{C} \mathrm{NMR}$ $\left(\mathrm{CDCl}_{3}, 125 \mathrm{MHz}\right): \delta=14.0,14.4\left(2 \mathrm{q}, \mathrm{CH}_{2} \mathrm{CH}_{3}\right), 20.4\left(\mathrm{q}, 8-\mathrm{CH}_{3}\right), 23.4$ (t, C-6), 39.0 (t, C-7), 46.4 (t, C-5), 47.3 (d, C-1), 58.9, 61.1 (2t, $\mathrm{CH}_{2} \mathrm{CH}_{3}$ ), 70.1 (s, C-8), 74.8 (d, C-8a), 98.1 (s, C2), 150.1 (d, C-3), 165.6, 174.4 (2s, CO); IR (KBr): v = $3445 \mathrm{~cm}^{-1}(\mathrm{OH}), 3075$ (=CH), 2980$2860(\mathrm{CH}), 1720(\mathrm{CO}), 1655,1580(\mathrm{C}=\mathrm{C})$; MS (EI, $\left.80{ }^{\circ} \mathrm{C}, 80 \mathrm{eV}\right): \mathrm{m} / z(\%)=297\left[\mathrm{M}^{+}\right](8)$, $279\left[\mathrm{M}^{+}-\mathrm{H}_{2} \mathrm{O}\right](6), 252\left[\mathrm{M}^{+}-\mathrm{C}_{2} \mathrm{H}_{5} \mathrm{O}\right]$ (4), $224\left[\mathrm{M}^{+}-\mathrm{C}_{4} \mathrm{H}_{5} \mathrm{O}_{2}\right]$ (100); $\mathrm{C}_{15} \mathrm{H}_{23} \mathrm{NO}_{5}$ (297.4) calcd.: C 60.59, H 7.79, N 4.71; found: C 60.50, H 7.51, N 4.59. 
$\frac{0}{n}$ 